\title{
Epidemiology of Gunshot-Related Injuries in NYC Emergency Departments from 2004-2014
}

\author{
Mansi Agarwal*, Nimi Idaikkadar and Don Weiss \\ New York City Department of Health and Mental Hygiene, Long Island City, NY, USA
}

\section{Objective}

To describe epidemiological characteristics of gunshot-related injuries in New York City (NYC) using syndromic surveillance data

\section{Introduction}

Syndromic surveillance has demonstrated utility for situational awareness for non-infectious conditions, including tracking and monitoring gun-related violence and homicides ${ }^{1}$. While New York State reports an overall decrease in gun-related crimes ${ }^{2}$, in NYC identifying smaller scale aberrations of gunshot-related violence can prompt more efficient response by city groups. We examined the temporal and borough-level characteristics of gunshot-related emergency department (ED) visits in NYC.

\section{Methods}

The NYC syndromic surveillance system captures ED visits from 49 hospitals on a daily basis. Chief complaints for each visit from January 1, 2004 through July 31, 2014 were scanned for gunshot wounds. Gunshot-related visits were identified by scanning chief complaints for the following keywords - "BULLET", "GSW", "GUN", "SHOT", "FIREARM", "RIFLE", and ICD9 code "E922.9." The scan algorithm was designed to include common misspellings and to exclude other types of shot-related complaints, such as vaccination and $\mathrm{BB}$ guns.

We calculated relative risks and 95\% confidence intervals for gunshot-related ED visits compared with other ED visits for temporal, spatial and weather-related characteristics. Hour of day was categorized into 3 categories, night ( $8 \mathrm{pm}-4 \mathrm{am})$, morning (4am$12 \mathrm{pm})$, and afternoon (12pm-8pm). A 4-level categorical variable for season and a binary variable for weekend were used to examine seasonal and day-of-week effects. Borough of patient residence was examined to identify spatial variation in gunshot-related injuries. Average daily temperature was categorized into a binary variable with $\geq 75^{\circ} \mathrm{F}$ classified as hot and the analysis was restricted to summer months of June, July, and August.

\section{Results}

There were 16,013 gunshot-related injuries identified during our study period. Men accounted for $91 \%$ of the visits but only $55 \%$ of all other ED visits. The median age of the patients was 24 years (mean 27 years; range 2 to 95 years).

From 2004 to 2014, NYC EDs have seen a 60\% decline in the annual proportion of gunshot-related visits. Visits were elevated in summer months $(\mathrm{RR}=1.73,95 \% \mathrm{CI}=1.66-1.81)$ as compared with winter months, with smaller but still significantly higher elevations in spring $(\mathrm{RR}=1.12,95 \% \mathrm{CI}=1.07-1.18)$ and fall $(\mathrm{RR}=1.35,95 \% \mathrm{CI}=$ 1.28-1.41). Gunshot-related visits were elevated at night as compared with afternoons $(\mathrm{RR}=3.12,95 \% \mathrm{CI}=3.01-3.24)$ and on weekends as compared with weekdays ( $\mathrm{RR}=1.86,95 \% \mathrm{CI}=1.80-1.92)$. In the summer, average daily temperature was also found to be a potential risk factor, with hotter days $\left(\geq 75^{\circ} \mathrm{F}\right)$ having higher rates of visits as compared with cooler days $\left(<75^{\circ} \mathrm{F}\right)(\mathrm{RR}=1.09,95 \% \mathrm{CI}=1.05-1.13)$.

Overall, there has been a decline in gunshot-related ED visits in all boroughs from 2004 to 2014, with Brooklyn experiencing a 50\% decrease in the annual proportion of gunshot-related visits. There was a significant association between borough and gunshot related visits. Compared with Manhattan, Brooklyn had the highest rates of gunshot-related visits $(\mathrm{RR}=2.07,95 \% \mathrm{CI}=1.97-2.18)$ followed by the Bronx $(\mathrm{RR}=1.12,95 \% \mathrm{CI}=1.06-1.18)$, Staten Island $(\mathrm{RR}=1.12$, $95 \% \mathrm{CI}=1.01-1.24)$, and Queens $(\mathrm{RR}=0.96,95 \% \mathrm{CI}=0.90-1.02)$.

\section{Conclusions}

Syndromic surveillance identified temporal and spatial patterns in gunshot related visits that are comparable to findings from the New York Police Department, suggesting that ED syndromic data have potential to be an accurate near-real time tracking system of gun-related injuries. Syndromic data may not capture all fatal gunrelated violence but do include patients who were dead on arrival. As gunshot-related injuries are typically emergencies, patients may be using other health care facilities for these visits. Therefore, ED data can be used to evaluate and improve interventions targeting gunrelated injuries. Continued monitoring of gunshot-related visits can be beneficial in identifying local hot spots in gun violence around NYC for an efficient public health response by health professionals, law enforcement, policy makers, and community-based organizations.

\section{Keywords}

Gunshot-related injuries; syndromic surveillance; NYC

\section{References}

1. Zeoli, AM, Pizarro, JM, Grady, SC, Melde, C (2012). Homicide as Infectious Disease: Using Public Health Methods to Investigate the Diffusion of Homicide. Justice Quarterly 31:3, 609-632.

2. Division of Criminal Justice Services. Office of Justice Research and Performance (2014). Crime, Arrest and Firearm Activity Report: Data Reported through June 30, 2014. 4 Aug. 2014. Accessed 15 Aug. 2014.

\author{
*Mansi Agarwal \\ E-mail: magarwal@health.nyc.gov
}

